\title{
An Adaptive Wavelet based Fusion Approach for Efficient Image Restoration
}

\author{
Simpy Kumari \\ M.Tech Scholar \\ Sagar Institute of Science \& Technology, Bhopal \\ Madhya Pradesh, India
}

\author{
Paresh Rawat, PhD \\ Professor and Head \\ Sagar Institute of Science \& Technology, Bhopal \\ Madhya Pradesh, India
}

\begin{abstract}
Nowadays lots of work is going to be done on the field of image fusion and also used in various application such as medical imaging and multi spectra sensor image fusing etc. For fusing the image various techniques has been proposed by different previous works such as wavelet transform, IHS (Intensity, Hue and Saturation) and Principal Component Analysis (PCA), based methods etc. In this paper literature of the image fusion is discussed with implementation using wavelet transform used for the specific application as in the image restoration field. Using Image fusion may improve the perceptual quality of the restored images. Usually image deblurring methods are used at the front end for restoration and then image fusion is used for improving the visual quality. Paper uses three de-blurring technique to blindly restoring the image then use statistical parameters for adopting the best fused images out of various hybrid fusion results. Performance is tested on images with distinct features.
\end{abstract}

\section{Keywords}

Image Restoration, Image Fusion, Discrete Wavelet Transform, Blind de-convolution, Wiener filter, Entropy

\section{INTRODUCTION}

Fusion imaging is one of the most contemporary, precise and useful diagnostic techniques in medical imaging today. The new skill has made a clear difference in patient care by compressing the time between diagnosis and treatment. Image fusion is the progression by which two or more images are combined into a single image retaining the important features from each of the original images. Image fusion mingles absolutely registered images from numerous sources to fabricate a high quality fused image with spatial and spectral information. So many image fusion methods have been developed from the past to now such as: the IHS [3], DCT [7], DWT [4], DT CWT [6] and PCA [3] methods etc. These methods functions under spatial domain $\&$ have proved to be flourishing in computer vision, robotics, satellite and medical image fusion applications.

\subsection{WHY IMAGES ARE BLUR}

Blur is un-sharp image area caused by camera or subject movement, inaccurate focusing, or the apply of a slit that gives shallow depth of field. The Blur effects are filters that level transitions and decrease contrast by averaging the pixels next to hard edges of defined lines and areas where there are significant color transition.

In digital image there are 3 common types of Blur effects:

\subsubsection{Average Blur}

The Average blur is one of a number of tools you be able to use to eliminate noise and specks in an image. Use it when noise is nearby over the entire image. This type of blurring can be distribution in horizontal and vertical direction and can be spherical averaging through radius $\mathrm{R}$ which is evaluated by the formula.

$$
R=\sqrt{ } g 2+f 2
$$

Where: $g$ is the horizontal size blurring direction and $f$ is vertical blurring size direction and $\mathrm{R}$ is the radius size of the circular average blurring.

\subsubsection{Gaussian Blur}

The Gaussian Blur effect is a filter that blends a specific number of pixels incrementally, subsequent a bell-shaped curve. The blurring is intense in the midpoint and feathers next to the edge. Apply Gaussian Blur to an image when you want more control over the Blur effect.

\subsubsection{Motion Blur}

When a photograph is occupied of any moving objective or the imaging system itself is moving subsequently the degradation caused is motion blur. Motion blur cause significant degradation of the image. This is caused by the association of the entity relative to the sensor inside the camera. The motion blur occurs if any of the following condition persists,

\section{i. Moving Object captured with static camera, \\ ii. Static Object captured with camera in motion, \\ iii. Both Object and camera be motion,}

iv. Shutter movement, film is exposed in a camera by the movement of the shutter across the film plane.

The Motion Blur effect is a filter that makes the image appear to be moving by adding a blur in a specific direction. The motion can be controlled by angle or direction ( 0 to 360 degrees or -90 to +90$)$ and/or by distance otherwise concentration in pixels (0 to 999), based on the software used.

\subsection{IMAGE DE-BLURRING MODEL}

Now let's pass on to more formal and scientific description of these blurring and restoration processes. We will consider only grayscale images, supposing that for processing of a fullcolor image it is enough to repeat all required steps for each of the RGB color channels. Let's introduce the following definitions:

$\mathrm{f}(\mathrm{x}, \mathrm{y})$ - source image (non-blurred)

$\mathrm{h}(\mathrm{x}, \mathrm{y})$ - blurring function

$\mathrm{n}(\mathrm{x}, \mathrm{y})$ - additive noise

$\mathrm{g}(\mathrm{x}, \mathrm{y})$ - blurring result image

We will form the blurring process model in the following way:

$$
g(x, y)=\square(x, y) * f(x, y)+n(x, y)
$$

The task of restoration of a blurred image consists in finding the best approximation $\mathrm{f}^{\prime}(\mathrm{x}, \mathrm{y})$ to the source image. Let's 
consider each component in a more detailed way. As for functions $\mathrm{f}(\mathrm{x}, \mathrm{y})$ and $\mathrm{g}(\mathrm{x}, \mathrm{y})$, everything is quite clear with them. But as for $\mathrm{h}(\mathrm{x}, \mathrm{y})$ I need to say a couple of words - what is it? In the process of blurring the each pixel of a source image turns into a spot in case of defocusing and into a line segment (or some path) in case of a usual blurring due to movement. Or we can say otherwise, that each pixel of a blurred image is "assembled" from pixels of some nearby area of a source image. Every one of those cover one another, which reality results in a blurred image. The rule, as indicated by which one pixel winds up spread, is known as the blurring capacity. Three sorts of wavelets utilized in the image fusion are Symmetrical; Bi-symmetrical and A-trous (Nonorthogonal).The image fusion strategy dependent on wavelet change has great spatial and ghastly distinction however has restricted directivity to manage the images having bended shapes. The image fusion is ordered into three level first pixel level second Attribute level and third Decision level.

a) Pixel Level Fusion It delivers a fused image in which data content related with every pixel is closed from an arrangement of pixels in source images. Fusion at this level can be complete either in spatial or in recurrence area. Nonetheless, pixel level fusion may direct to differentiate decrease.

b) Attribute Level Fusion Property level fusion requires the extraction of striking attributes which are relying upon their surroundings, for example, pixel powers, edges or surfaces. These similar to trait from the info images are fused. This fusion level can be utilized as a methods for making valuable amalgamated traits. The fused image can likewise be utilized for grouping or identification.

c) Decision Level Fusion Choice level is a prevalent level of fusion. Information images are handled autonomously for data mining. The acquired data is then joined applying choice standards to stress far reaching translation The advantage of multi-sensor image fusion comprises:

i Improved reliability - The fusion of different measurements can diminish noise and consequently develop the steadfastness of the measured quantity.

ii Robust system performance - Redundancy in various measurements can help in systems stoutness. In case one or more sensors fail or the performance of a meticulous sensor deteriorates the system can depend on the other sensors.

iii. Compact representation of information - Fusion leads to condensed representations. For example, in remote sensing, instead of storing imagery from numerous spectral bands, it is moderately more proficient to store the fused information.

iv. Extended range of operation - Multiple sensors that function under different operating conditions can be deployed to expand the effective range of operation. For example, different sensors can be used for day/night operation.

v. Extended spatial and temporal coverage - Joint information from sensors that diverge in spatial resolution can increase the spatial coverage. The identical is true for the secular dimension

vi. Reduced uncertainty - Joint information from several sensors can diminish the vagueness associated with the sensing or decision process.

The image fusion process is characterized as social affair all the imperative data from numerous images and their incorporation into less images, for the most part a solitary one. This single image is more instructive and precise than any single source image, and it comprises of all the essential data. The reason for image fusion isn't just to lessen the measure of information yet in addition to develop images that are more suitable and justifiable for the human and machine discernment.

1. In computer vision, Multi-sensor Image fusion is the process of combining relevant information from two or more images into a single image.

2. The resulting image will be more informative than any of the input images.

3. In remote sensing applications, the increasing availability of space borne sensors gives a motivation for different image fusion algorithms. Several situations in image processing require high spatial and high spectral resolution in a single image.

A large portion of the accessible gear isn't equipped for giving such information convincingly. Image fusion strategies permit the combination of various data sources. The fused image can have reciprocal spatial and ghostly goals qualities. Be that as it may, the standard image fusion systems can mutilate the unearthly data of the multispectral information while combining.

In satellite imaging, two sorts of images are accessible. The panchromatic image obtained by satellites is transmitted with the most extreme goals accessible and the multispectral information are transmitted with coarser goals. This will more often than not be two or four times lower. At the beneficiary station, the panchromatic image is converged with the multispectral information to pass on more data.

Numerous strategies exist to perform image fusion. The simple fundamental one is the high pass sifting procedure. Later procedures depend on Discrete Wavelet Change, uniform judicious channel bank, and Laplacian pyramid. A.

\subsubsection{Multi-Focus Image Fusion}

Multi-focus image fusion

Multi-center image fusion is utilized to gather valuable and vital data from info images with various center profundities with the end goal to make a yield image that in a perfect world has all data from information images. In visual sensor network (VSN), sensors are cameras which record images and video successions. In numerous utilizations of VSN, a camera can't give an impeccable outline including all points of interest of the scene. This is a result of the restricted profundity of center exists in the optical focal point of cameras Subsequently, simply the question situated in the central length of camera is engaged and cleared and alternate parts of image are blurred. VSN has a capacity to catch images with various profundity of centers in the scene utilizing a few cameras. Because of the huge measure of information created by camera contrasted with different sensors, for example, weight and temperature sensors and some confinement, for example, constrained band width, vitality utilization and handling time, it is fundamental to process the neighborhood input images to diminish the measure of transmission information. The previously mentioned reasons stress the important of multi-center images fusion. Multi-center image fusion is a procedure which consolidates the info multi-center images into a solitary image including exceedingly vital data of the information images and it's more precise clarification of the scene than each and every information image. 


\subsubsection{Standard Image Fusion Methods}

Image fusion techniques can be extensively characterized into two gatherings - spatial space fusion and change area fusion.

The fusion strategies, for example, averaging, Brovey technique, main segment investigation (PCA) and HIS based strategies fall under spatial area approaches. Another imperative spatial area fusion strategy is the high pass separating based procedure. Here the high recurrence points of interest are infused into up examined form of MS images. The burden of spatial area approaches is that they deliver spatial mutilation in the fused image. Unearthly contortion turns into a negative factor while go for further handling, for example, arrangement issue. Spatial contortion can be exceptionally all around dealt with by recurrence space approaches on image fusion. The multi-goals examination has turned into an extremely valuable instrument for dissecting remote detecting images. The discrete wavelet change has turned into an extremely helpful instrument for fusion. Some other fusion strategies are likewise there, for example, Laplacian pyramid based, bend let change based and so on. These techniques demonstrate a superior execution in spatial and ghastly nature of the fused image contrasted with other spatial strategies for fusion.

The images utilized in image fusion should as of now be enlisted. Misregistration is a noteworthy wellspring of mistake in image fusion. Some outstanding image fusion techniques are:

- $\quad$ High pass filtering technique

- HIS transform based image fusion

- $\quad$ PCA based image fusion

- Wavelet transform image fusion

- Pair-wise spatial frequency matching

\section{LITERATURE SURVEY}

Different techniques are proposed in writing for upgrading the images, for example, strategies utilizing the image histogram, local region based improvement, and change area strategies. In this segment paper audits a portion of the significant strategies for upgrading the true color images.

J. Jiao and W. Lingda,In request to viably join the ghastly data of the multispectral (MS) image with the spatial subtle elements of the panchromatic (Container) image and enhance the fusion quality, a fusion strategy dependent on morphological administrator and enhanced heartbeat coupled neural system $(\mathrm{PCNN})$ in blended multi-scale $(\mathrm{MM})$ area is proposed. Right off the bat, the MS and Dish images are decayed by non sub inspected shear let change (NSST) to low-and high-recurrence coefficients, separately; besides, morphological channel based power balance (MFIM) innovation and stationary wavelet change (SWT) are connected to the fusion of the low-recurrence coefficients; an enhanced PCNN demonstrate is utilized to the fusion of the high-recurrence coefficients; thirdly, the last coefficients are reproduced with converse NSST. The exploratory outcomes on Speedy Fledgling satellite show that the proposed technique is better than five different sorts of conventional and mainstream strategies: HIS, PCA, SWT, NSCT-PCNN and NSST-PCNN. The proposed technique can enhance the spatial goals adequately while keeping up the ghostly data well. The test results demonstrate that the proposed technique outflanks alternate strategies in visual impact and goal evaluations.[1]

Z. Fan, L. Yan, On account of the poor lighting conditions during the evening, noticeable images are frequently fused with comparing infrared images for improvement of the scenes in night vision. In this paper, a novel image fusion calculation is exhibited for fusion of obvious image and infrared image with various goals. The multi-goals image fusion is carried on by the accompanying five stages:

1) Distinctive goals images are changed into a similar goals by utilization of wavelet change;

2) to upgrade the perceivability of dim locale content in the decayed noticeable image, a novel night-vision upgrade technique is introduced dependent on guided channel; 3) the infrared image data is infused into the unmistakable image through a multi-scale fusion approach dependent on guided channel; 4) converse wavelet change is utilized to accomplish the fusion image; 5) to additionally enhance the perceivability of the fusion image, it is improved by utilization of a versatile upgrade method.[2]

C. C. Chaithra, N. L. Taranath, Image fusion is the way toward consolidating at least two important data into one image. The came about image will have more logical than unique images. Multispectral image (MS) is gotten from satellite, multispectral image having rich phantom data and low spatial goals. MS have less data which isn't reasonable for remote sensor application. Panchromatic (Dish) image is one of the sorts of satellite images. Dish images have more otherworldly data yet low spatial data. In remote detecting application more spatial and ghastly data is required, so blending MS and Skillet will result in rich spatial and otherworldly image. Numerous fusion calculations are bolstered to meld MS and Skillet. A few methods are primary part examination, discrete wavelet change, pixel-level image fusion and multi-sensor image fusion. Subjective investigation decides the execution of fused image by examination between unique image and came about fused image. Some subjective measurements are assessed utilizing Root mean square blunder (RMSE), Relative worldwide dimensional amalgamation mistake (ERGAS), Quality factor (Q4), Cross relationship (CC) and Unearthly point mapper (SAM). This work surveys about different fusion strategies in remote sensor and quality metrics.[3]

P. K. R. Yelampalli, another nearby element descriptor recursive Daubechies design (RDbW) is created by characterizing and encoding the Daubechies wavelet decayed center-nalyzin pixel relationship in the neighborhood surface. RDbW highlights are connected in spatial arrangement (enrollment) of multimodal restorative images utilizing a Procrustes investigation (Dad)- based relative change work and the enlisted images are additionally fused by utilizing a wavelet-based fusion technique. A lot of investigations is led and the enlistment and fusion exactness of the proposed highlight descriptor is contrasted and the unmistakable existing techniques, for example, neighborhood twofold examples (LBP), nearby tetra design (LTrP), neighborhood askew outrageous example (LDEP), and neighborhood slanting Laplacian design (LDLP). Test results demonstrate the present enlistment strategy enhances the normal enrollment precision by $38,47,71$, and $76 \%$ as opposed to LDLP, LDEP, LTrP, and LBP, separately. Further, the fusion aftereffects of the present methodology show a normal change in entropy by $11 \%$, standard deviation by $6 \%$ edge quality by $12 \%$, sharpness by $23 \%$, and normal slope by $16 \%$ when 
contrasted and all other element descriptors utilized for enlisting the images. Ideas introduced here can be utilized broadly in nalyzing the consolidated data present in multimodal medicinal images.[4]

Y. Tong and J. Chen Most imaging frameworks have a restricted profundity of-field in the sensor organizes that comprise of different visual sensors. Because of various protest separations, few out of every odd question can be obviously imaged by a solitary sensor. This paper proposes a multi-center image fusion calculation in sensor systems. The calculation joins the benefits of multi-scale examination and image stage investigation. It utilizes dynamic window and stage extend change (PST) to remove the engaged locales of each image precisely and performs multi-goals investigation of images through the non-sub-tested shear let change. At that point, the images are fused by the nearby standard deviation of PST highlight maps. The consequences of the reproduction tests demonstrate that our calculation is compelling and outflanks some cutting edge algorithms.[5]

\section{PROPOSED IMAGE FUSION METHOD}

Wavelet change is first performed on source images. At that point a fusion choice guide is produced dependent on an arrangement of fusion rules.

At that point melded wavelet coefficient guide can be built from the wavelet coefficients of the source images as indicated by the fusion choice guide. At long last the melded image is acquired by playing out the backwards wavelet change. The DWT based systems wound up prominent due to their multi-goals properties. The primary issue in wavelet fusion procedure is the determination of disintegration level and the ideal wave channel

\subsection{Pixel Based Fusion Rules}

It is a intensity biased image fusion method. In which we straightforwardly take a shot at the intensities of individual pixels. Let $\mathrm{A}(\mathrm{x}, \mathrm{y})$ and $\mathrm{B}(\mathrm{x}, \mathrm{y})$ are images to be combined, the decayed low recurrence sub images of $\mathrm{A}(\mathrm{x}, \mathrm{y})$ and $\mathrm{B}(\mathrm{x}$, $y)$ are $1 \mathrm{AJ}(\mathrm{x}, \mathrm{y})$ and $\mathrm{lBJ}(\mathrm{x}, \mathrm{y})$. Deteriorated high recurrence sub images of $A(x, y)$ and $B(x, y)$ are $h A j, k(x, y)$ and $h B j, k(x$, $y)$. ( $j$ and $k$ is the parameter of goals, where $j=1,2,3 \ldots . J$ for each $\mathrm{j}$, and $\mathrm{k}=1,2,3 \ldots \mathrm{k}$ for each $\mathrm{k}$ ) There are distinctive pixel level fusions techniques like as:

\subsection{Pixel Averaging (Method1)}

All the four sub bands of the fused image $\mathrm{F}$ is simply acquired by averaging the wavelet coefficients of source images A \& B.

$$
F_{j k}=\left(A_{j k}+B_{j k}\right) / 2 \& F_{J}=(l A j+l B j) / 2
$$

\subsection{Proposed adaptiveFusion techniques.}

In this paper the wavelet constructed fusion is actualized with respect to the first and the improved images for therapeutic imaging applications. the min, max and averaging pixel level fusion rules are embraced dependent on the entropy examination the correlation of the entropy of the blurred images utilizing each of the three techniques are made and the best entropy is received as the versatile fusion rules. The examinations of wavelet decay for the first and improved CLAHE images are appeared in the Figure 3. The information therapeutic image and upgraded image is fused utilizing the three pixel level fusion rules Viz. Pixel level Maxima, Pixel Level Minima and Pixel Level Averaging or mean.

The calculation of image fusion utilizing DWT portrayed in the following advances
1. Size of sources of info images: Given a two dimensional images (precedent, image An, image B) it is important to change over it into a similar size an intensity of two square structures.

2. Calculation of two measurements DWT: In this progression, the two dimensional Discrete Wavelet Change ought to be connected to the resized two dimensional images.

3. Fusion run: The most utilized of image fusion run utilizing wavelet change is greatest determination; analyze the two coefficients of DWT of the two images and select the most extreme between. While the low pass sub band is an estimation of the info image, the three detail sub bands pass on data about the detail parts in flat, vertical and askew bearings. Diverse blending methodology will be connected to estimate and detail sub bands. Low pass sub band will be consolidated utilizing straightforward averaging tasks since they both contain approximations of the source images.

4. Backwards discrete wavelet changes: After chose the fused low recurrence and high recurrence bands, fused coefficient is reproduced utilizing the Converse quick discrete wavelet change to get the fused image which speak to the new image.

\subsection{PROPOSED ADAPTIVE FUSION}

In this adaptive method, first input image is de-blurred using the Blind de-convolution, Lucy Richardson (LR), and Wiener Filter method of image restoration. Then the restored images are fused together using wavelet fusion techniques. In this technique these fused images are wavelet decomposed up to level $\mathrm{N}$ using Discrete wavelet transform. The Low pass and high pass sub-bands are then fused utilizing distinctive pixel level fusion strategies is executed for creating various mixture combined images. At that point the backwards wavelet change is performed to get full size melded images. The after-effects of pixel level intertwined images are thought about dependent on the entropy examination. The after-effects of greatest entropy are at last chosen as the last re-established image having most extreme visual substance. Proposed versatile fusion technique embraces mix of the best de-obscuring strategy and pixel level fusion rules for image reclamation dependent on entropy augmentations utilizing the tri arrange Entropy correlation as given here.

E1 $>$ E2 \& E1>E3 Image ${ }_{\text {out }}=$ Pixel level Maxima

$$
\begin{aligned}
& \text { E1 }<\text { E2 \& E2 }>\text { E3 then Image }{ }_{\text {out }}=\text { Pixel level Minima } \\
& \text { E3 }>\text { E1 \& E3 }>\text { E1 then Image }{ }_{\text {out }}=\text { Pixel level Averaging }
\end{aligned}
$$

It is observed that adaptive fusion rule selection the efficiency of the medical image enhancement is improved since now the fusion results are environment independent.

\section{RESULT AND DISCUSSION}

Implementation of image fusion using wavelet is performed in MATLAB software. In figure 1 clock and flower image is taken to perform.
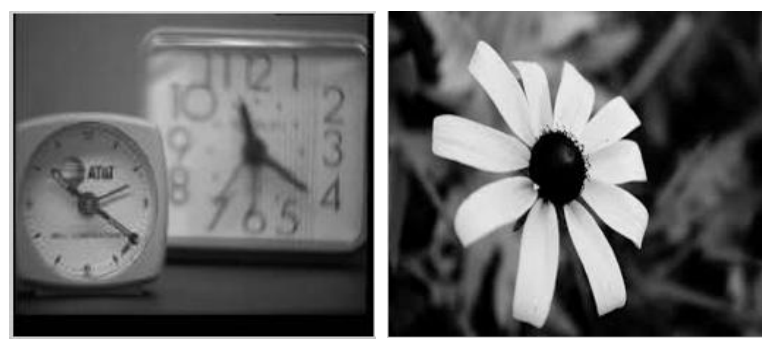
Figure 1: Original Clock and Flower Image.

In figure 2, first level of discrete image transform is showing, where image frame is divided in 4 parts.
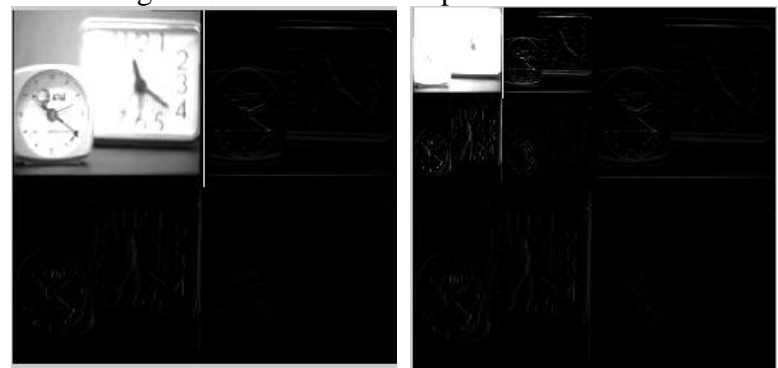

(a)1st level decomposition (b) 2nd level Decomposition Figure 2 DWT decomposition image of flower image

In Figure 2 b) presents second level of discrete image transform is showing, where image frame is divided in 4 parts and out of all part again one part is divided in 4 parts.

In Figure 3 minima and maxima, average and adaptive fused clock image is showing.
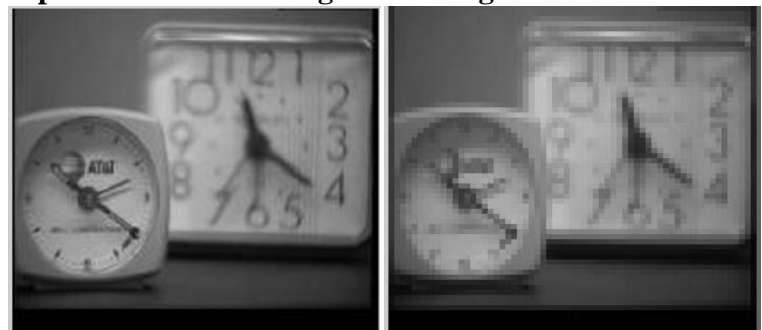

(a) Minima fused image (b) Maxima fused image
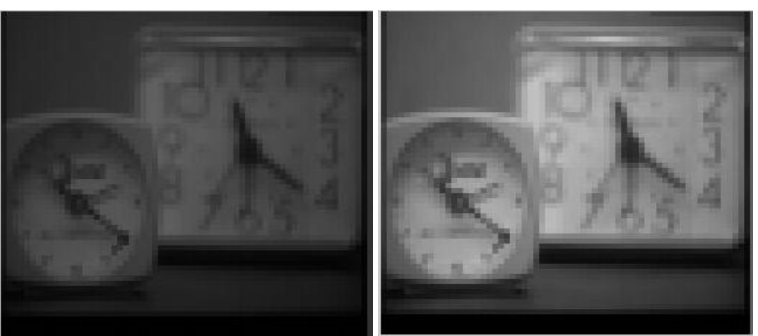

c) Average fused image

(d) Finally adaptive fused image Figure 3: Fusion results for the Clock image

It can be observed from the Figure 3 that the adaptive fusion method may improve the features and numbers in the images. In Figure 4 first level of discrete image transform is showing, where image frame is divided in 4 parts.
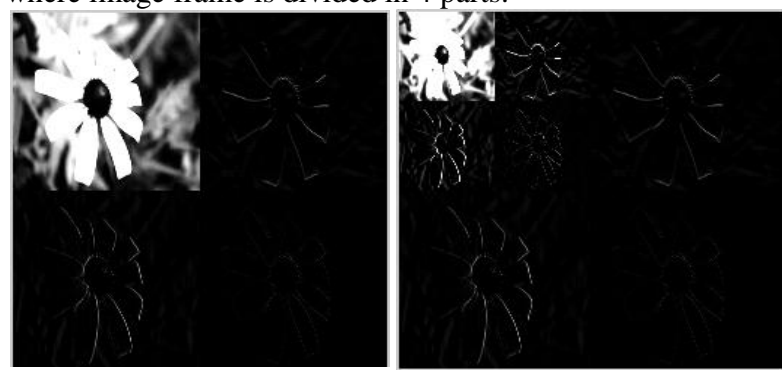

a) $1^{\text {st }}$ level decomposition b) $2^{\text {nd }}$ level Decomposition

Figure 4 : DWT decomposition image of flower image

In figure 4, second level of discrete image transform is showing, where image frame is divided in 4 parts and out of all part again one part is divided in 4 parts.

Table 1: Comparison of the entropy for fusion methods

\begin{tabular}{|l|l|c|c|c|c|c|}
\hline $\begin{array}{l}\text { Sr. } \\
\text { No. }\end{array}$ & Images & Original image & Minima Fused & Maxima Fused & Average Fused & $\begin{array}{c}\text { Proposed } \\
\text { Adaptive Fusion }\end{array}$ \\
\hline 1 & Clock & 7.3645 & 0.2630 & 7.2765 & 6.3522 \\
\hline 2 & Flower & 7.4241 & 0.4018 & 7.4384 & 6.5034 \\
\hline
\end{tabular}

Figure 5, minima and maxima fused flower image is shown. An average fused image of original image is showing and finally adaptive fused is showing in the Figure 5. C) and Figure 5 d) respectively. It can be observed from the results that our proposed adaptive fusion method gives the best result in terms of the visual quality of the images.
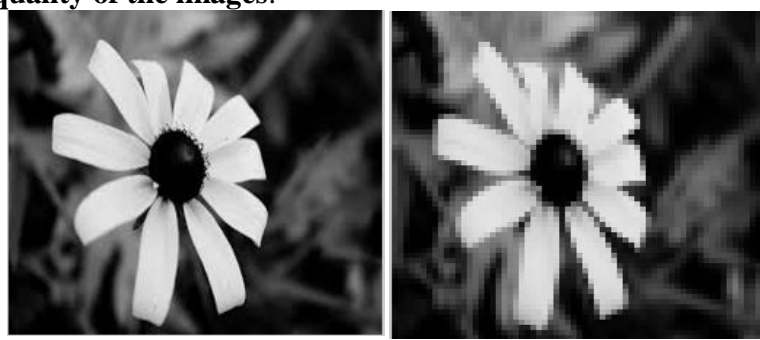

(a) Minima fused image (b) Maxima fused image
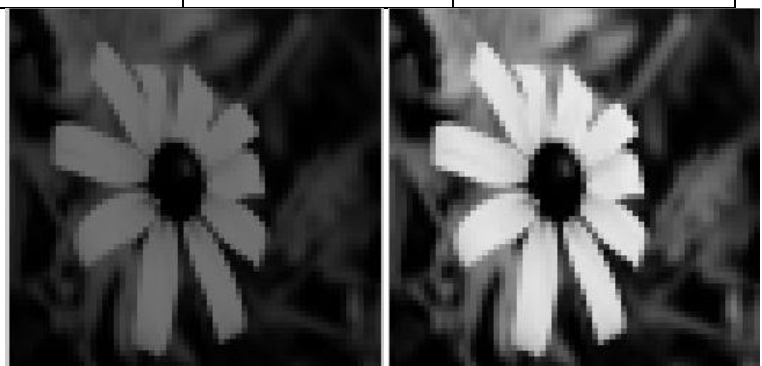

c) Average fused image

(d) finally adaptive fused image Figure 5: Fusion results for the Flower image

It can be observed from Table 1 that for Wiener filter the pixel level maxima is adopted with maximum entropy.

\section{CONCLUSION}

The wavelet transform is a standout amongst the most proficient ways to deal with concentrate the highlights by the change and decay process however this technique isn't productive to hold the edge data. In this paper writing investigation of the fusion methods is portrayed with usage of wavelet based image fusion. Image information fusion has turned into a significant instrument used to incorporate the best qualities of every sensor information associated with the handling. To be sure, the above procedures, strategy and applications are utilized for scientists, to allude the image 
fusion. The fusion quality regularly relies on the client's understanding, the fusion strategy, and upon the informational collection being fused.

\section{REFERENCES}

[1]. J. Jiao and W. Lingda, "Fusion of Panchromatic and Multispectral Images via Morphological Operator and Improved PCNN in Mixed Multiscale Domain," 2018 10th IAPR Workshop on Pattern Recognition in Remote Sensing (PRRS), Beijing, China, 2018, pp. 1-11.

[2]. Z. Fan, L. Yan, Y. Xia, M. Fu and B. Xiao, "Fusion of multi-resolution visible image and infrared images based on guided filter," 2018 37th Chinese Control Conference (CCC), Wuhan, China, 2018, pp. 4449-4454.

[3]. C. C. Chaithra, N. L. Taranath, L. M. Darshan and C. K. Subbaraya, "A Survey on Image Fusion Techniques and Performance Metrics," 2018 Second International Conference on Electronics, Communication and Aerospace Technology (ICECA), COIMBATORE, India, 2018, pp. 995-999.

[4]. P. K. R. Yelampalli, J. Nayak and V. H. Gaidhane, "Daubechies wavelet-based local feature descriptor for multimodal medical image registration," in IET Image Processing, vol. 12, no. 10, pp. 1692-1702, 102018.

[5]. Y. Tong and J. Chen, "Multi-Focus Image Fusion Algorithm in Sensor Networks," in IEEE Access, vol. 6, pp. 46794-46800, 2018.

[6]. G Easley, D Labate, W Q. Lim, "Sparse directional image representations using the discrete shearlet transform", Applied \& Computational Harmonic Analysis, vol. 25, no. 1, pp. 25-46, 2008.

[7]. X. Xing, Research on the image fusion algorithm based on no-subsampled shearlet transform, Jilin University, 2014.

[8]. E. Li, Image mosaic and fusion technology of large field view multi-spectral camera, Changchun Institute of
Optics, Fine Mechanics and Physics, Chinese Academy of Sciences, 2015.

[9]. E P. Blasch, "Biological information fusion using a PCNN and belief filtering", Proceedings of the International Joint Conference on Neural Networks., vol. 4, pp. 2792-2795, 1999.

[10]. Y Liao, W L Huang, L Shang et al., "Image fusion based on Shearlet and improved PCNN", Computer Engineering and Applications, vol. 50, no. 2, pp. 142146, 2014.

[11].B Qu, J W Yan, H Z. Xiao, "Image Fusion Algorithm Based on Spatial Frequency-Motivated Pulse Coupled Neural Networks in Nonsubsampled Contourlet Transform Domain", Acta Automatica Sinica, vol. 34, no. 12, pp. 1508-1514, 2008.

[12].P Jiang, Q Zhang, J. Li, "Fusion algorithm for infrared and visible image based on NSST and adaptive PCNN", Laser \& Infrared, vol. 44, no. 1, pp. 108-113, 2014.

[13]. K Zhang, M Wang, S Yang et al., "Fusion of Panchromatic and Multispectral Images via Coupled Sparse Non-Negative Matrix Factorization", IEEE Journal of Selected Topics in Applied Earth Observations \& Remote Sensing, vol. 99, pp. 1-8, 2016.

[14]. R Restaino, G Vivone, M D Mura, J. Chanussot, "Fusion of MultiSpectral and Panchromatic Images Based on Morphological Operators", IEEE Transactions on Image Processing, vol. 25, no. 6, pp. 2882-2895, 2016.

[15].Paresh Rawat, Sapna Gangrade, Pankaj Vyas" Implementation of Hybrid Image FusionTechnique Using Wavelet Based Fusion Rules" International Journal of Computer Technology and Electronics Engineering (IJCTEE) 